\title{
AN OVERVIEW OF SIMULATION, MODELING, AND ACTIVE CONTROL OF FLOW/ACOUSTIC RESONANCE IN OPEN CAVITIES
}

\author{
Tim Colonius * \\ Division of Engineering and Applied Science \\ California Institute of Technology \\ Pasadena, CA 91125
}

\begin{abstract}
An overview of some recent advances in simulation, modeling, and control of flow/acoustic resonance in flows over open cavities is provided. A wide variety of experiments utilizing differing actuator concepts, with both open and closed-loop control, have shown that significant attenuation of tones and broadband noise is possible. These are discussed in connection with recent advances in theoretical modeling and numerical simulation. Such work may ultimately provide accurate low-order models of cavity resonance that are suitable for robust and effective control over a wide range of flow conditions. Several areas that will require further advances in our understanding of the flow physics are highlighted.
\end{abstract}

\section{INTRODUCTION}

The purpose of this paper is to provide an overview of some recent advances in simulation, modeling, and control of flow/acoustic resonance in flows over shallow open cavities. Many of the results are of course relevant to similar flows, such as jet screech, edge tones, and jet impingement, that involve unstable shear layers in the presence of strong acoustic sources. An exhaustive survey of the previous literature, dating back to the mid 1950 's, on flow/acoustic instabilities, and cavity oscillations in particular, is not possible in a short paper, and some previous reviews should be consulted for a more complete view of the literature..$^{2,40,41}$ While an attempt has been made to cite the relevant work since the previous reviews, there are undoubtedly important omissions, for which the author apologizes in advance. The next section discusses the experimental attempts at noise suppression in the cavity. This also serves to motivate the discussion of modeling and simulation in the later sections, where we attempt to identify important issues that will require further study in order to implement effective control of cavity tones and broadband noise.

\footnotetext{
* Associate Professor of Mechanical Engineering, Member AIAA

Copyright (c) 2001 by the authors. Published by the American Institute of Aeronautics and Astronautics, Inc. with permission.
}

\section{SUPPRESSION OF CAVITY NOISE}

Recent work on the cavity problem has been motivated by the desire for noise control in open cavities on aircraft. From modest subsonic to supersonic speeds, internal weapons bays are subjected to intense internal sound pressure levels (levels in excess of $160 \mathrm{~dB}$ are not uncommon) and these can damage stores, fatigue nearby surfaces and components, and adversely affect store separation and accurate delivery of weapons. ${ }^{49,52}$ It has long been known that passive devices, such as spoilers and fences near the separation point, and trailing edge ramps can significantly reduce tone amplitudes. ${ }^{40}$ More recent examples of passive devices include pneumatic control, whereby pressure difference in the cavity are used to transport fluid along the cavity floor, ${ }^{9}$ small rods placed in crossflow just upstream of the cavity, ${ }^{51,58}$ and passive "resonance tubes". ${ }^{58}$ It has been observed ${ }^{37}$ that many of these devices are only effective over limited flow conditions, and can actually enhance tones at off-design conditions*.

These observations coupled with recent advances in the actuator technologies have thus served to focus interest on control strategies that are in some sense adaptable to varying flow conditions. Sarohia and Massier ${ }^{48}$ found that mass injection at the cavity base suppressed cavity tones. Experiments by Gharib ${ }^{19}$ on incompressible cavity flow demonstrated the feasibility of controlling cavity oscillations by external forcing of the cavity shear layer, achieving a $40 \%$ reduction in tone amplitude by exciting unstable Tollmein-Schlichting waves upstream of the cavity. Sarno and Franke $e^{45}$ achieved tone reduction with steady and pulsed mass injection through a slot just below the upstream cavity edge (both parallel and at 45 degrees inclination to the freestream) achieving some reduction in amplitude $(10 \mathrm{~dB})$ but not as great as that achieved with a static fence (spoiler) at the upstream edge. Computations ${ }^{32}$ at similar conditions (with steady and pulsed blowing) showed similar reductions. Sarno et

\footnotetext{
${ }^{*}$ Recently reported results on model scale weapons cavities ${ }^{58}$ suggest that passive devices that create flow disturbances at frequencies that are high compared to the cavity tones may reduce noise over a wider Mach number range
}

1 
$a l .{ }^{45}$ found that mass injection was inferior to amplitude reductions produced by a static fence (spoiler) at the upstream edge at the two (supersonic) Mach numbers they studied. Vakili and Gauthier ${ }^{63}$ reported much larger reductions $(27 \mathrm{~dB})$ of the dominant tone, with steady mass injection just upstream of the cavity. These studies all used fairly large mass flow rates, with blowing parameter $\rho_{b} U_{b} / \rho_{\infty} U_{\infty}$ on the order of 0.1 for the steady injection. Leading edge blowing was recently tested on a $20 \%$ scale model tests of a weapons bay in preparation for a potential F-111 flight test. ${ }^{22}$ At $\mathrm{M}=0.9$, tones with "full" bays showed reductions as high as $15 \mathrm{~dB}$ (blowing at $1 \mathrm{lbm} / \mathrm{sec}$ ), but had unexpected effects on the forces and pitching moments on the stores. Several other investigators ${ }^{33}, 49$ have suggested that pulsed blowing can achieve tone reductions with lower net mass flow rates than steady blowing. Williams and Fabris ${ }^{64,65}$ report successful attenuation of resonant modes with a zero net mass flux slot connected to an acoustically driven cavity ("unsteady bleed forcing").

Other devices that have been used to successfully suppress one or more cavity tones include piezoelectric flappers at the leading edge, ${ }^{6}$ piezoelectric wedges upstream of the cavity, ${ }^{29,58}$ miniature fluidic oscillators placed on the cavity floor, ${ }^{39}$ and powered resonance tubes. ${ }^{58}$

Particularly good suppression of tones and broadband noise was reported by Stanek et al. ${ }^{58}$ with highfrequency actuators (operating frequencies of several $\mathrm{kHz}$ ). Most effective were a powered resonance tube (that required roughly $0.4 \mathrm{lbm} / \mathrm{sec}$ air supply, but was thought to effect the flow primarily through acoustic forcing) and a simple, passive rod in crossflow. They provide evidence that these devices produce a similar effect on the cavity flow as has been achieved by Wiltse and Glezer ${ }^{66}$ in free shear flow experiments. That is, small-scale turbulence is directly excited by the device. The hypothesis is that such forcing accelerates the transfer of energy in the inertial range, essentially "starving" the lower frequency turbulence (and in the case of cavity, resonant modes) of energy.

Only a few of the aforementioned studies utilized feedback controllers in their experiments. The group at High Technology Corporation ${ }^{6}$ developed an adaptive disturbance rejection algorithm that includes a system identification procedure so that the controller can tune itself to achieve better performance (see ref. ${ }^{6}$ and references therein for details). The system was very effective in low Mach number tests. Williams and Fabris ${ }^{64}$ have developed a feedback controller that processes the sensor signal (a Kulite transducer located in the upstream wall) with a bandpass filter and phase shift. Successful suppression was achieved at Mach numbers as high as 0.55 . Shaw and Northcraft ${ }^{52}$ also performed closedloop control (presumably also by directly feeding back the pressure signal to their pulsed fluidic actuator). Finally, Kestens and Nicoud ${ }^{27}$ present an interesting numerical study that attempts to use feedback control of a loudspeaker to cancel the pressure fluctuations at a microphone location.

Aside from adapting to changing flow conditions, feedback control has the advantage that the required power levels to drive an actuator are typically greatly reduced after control is achieved. However, effective feedback control of flows is made difficult by, on one hand the flow's sensitivity to external disturbances, and on the other hand by the difficulty of establishing accurate system models that fit into the framework of modern control theory. A discussion of the relevant issues in a general context is presented in some recent reviews. ${ }^{1,18}$ Generally speaking, the better the understanding of the flow physics, the more likely it is that robust and efficient control strategies may be developed.

In the remainder of this paper, issues that are thought to be of important in establishing reliable low-order models of cavity resonance are presented. In section 3, we discuss the status of the prediction of the frequencies and amplitude of the resonant modes. Numerical simulations (section 4) provide a useful database for examining modeling issues, albeit at low to moderate Reynolds number, in the case of Direct Numerical Simulations (DNS) and Large Eddy Simulations (LES), or at higher Reynolds number, albeit with additional assumptions regarding the efficacy of the turbulence modeling, with the Reynolds Averaged Navier-Stokes (RANS). In addition, such simulations provide a database for modeling efforts that employ the POD/Galerkin projection (as well as other data reduction methods) in order to distill a low-order model, as discussed in section 5 .

\section{Flow Physics And Modeling}

A distinguishing feature of the type of flow/acoustic resonance considered here is the existence of a feedback loop involving (i) excitation of an unstable shear layer, (ii) generation of an unsteady irrotational field by interaction of the shear layer with a solid boundary (in the case of cavity, edge tones, or jet impingement) or a shock wave (in the case of screech) and (iii) the upstream influence of the irrotational field which provides for the further excitation of the instabilities in the shear layer, especially near the upstream edge. For incompressible flow, the upstream influence is instantaneous, while for compressible flow there is an acoustic delay. Resonance occurs when the phase change of a disturbance, at a given frequency, leads to constructive rein- 
forcement and, ultimately, saturation. Because of the coupling with flow instability, cavity resonant frequencies and amplitudes may depend on the flow speed, $U$, the boundary layer (momentum) thickness, $\theta$ just upstream of the cavity, and ambient density, $\rho_{\infty}$, viscosity, $\mu_{\infty}$, and sound speed, $a_{\infty}$. For the three dimensional rectangular cavity with length, $L$, depth, $D$, and breadth, $H$, there are thus 5 dimensionless parameters governing the flow $L / D, L / H, L / \theta, \operatorname{Re}_{\theta}=\frac{\rho_{\infty} U \theta}{\mu_{\infty}}, M=\frac{U}{a_{\infty}}$ as well as additional parameters and profiles that would specify the state of the boundary layer upstream of the cavity if it is turbulent. This can be distinguished from pure acoustic resonance where frequency selection depends only on the geometrical constructive reinforcement of acoustic reflections

The idea of such a feedback cycle was apparently first put forward by Powell ${ }^{38}$ in his work on edge-tones. Krishnamurty $^{31}$ identified acoustic tones associated with flow over open cavities. Rossiter ${ }^{43}$ performed an extensive set of experiments that identified a series of discrete frequencies of oscillation (modes) that may be present either solely or in combination, as a function of Mach number and length to depth ratio of the cavity. He used the idea of the feedback cycle for the cavity to develop a semi-empirical formula to predict the resonant frequencies:

$$
S t_{n}=\frac{f_{n} L}{U}=\frac{n-\alpha}{M+\frac{1}{\kappa}}, \quad n=1,2,3, \ldots
$$

where $S t_{n}$ is the Strouhal number corresponding to the $n$-th mode frequency, $f_{n}, \kappa$ is the average phase speed of the vortical disturbances, and $\alpha$ is an empirical constant, typically taken around 0.25 . Various refinements of this formula have been suggested $\left(e . g .{ }^{40}\right)$, for example to account for the higher sound speed inside the cavity at higher Mach numbers. Spectra from a large number of experiments over the years show reasonable agreement with equation (1), but with a significant amount of scatter. The scatter is particularly strong at low Mach numbers. This is likely due to an interaction with normal acoustic resonant modes of the rectangular cavity. $6,40,60,65$ Some experiments, e.g. ${ }^{47}$ however, continue to show good agreement with the Rossiter equation at very low Mach numbers. There does not yet appear to be any systematic way to detect if or when these low Mach number interactions will occur. At higher Mach numbers the scatter is less, and is probably due, in large part, to the variation of $\kappa$ due to different shear layer thickness in the various studies.

The data for the saturation amplitudes of the oscillating modes exhibit far greater scatter than the frequencies, even at very similar Mach numbers and cavity geome- tries. Undoubtedly this sensitivity is in part due to sensitivity of the amplification of disturbances in the shear layer, and changes to the spreading of the layer that result from the disturbances and incoming turbulence. Given the local spreading rate of the shear layer, or an average value over the cavity, the growth of disturbances in the shear layer, and their parametric variations, may be estimated using a quasi-parallel stability analysis of the shear layer in isolation (e.g. ${ }^{40}$ and refs therein). Sarohia, ${ }^{47}$ for example, predicts the dominant mode over a range of parameters by computing the integrated spatial amplification. Linear stability calculations are indeed valuable for understanding certain sensitivities of the amplitudes with respect to the flow conditions. Figure 1 shows the integrated amplification as the length and depth of the cavity are varied holding the boundary layer thickness at separation constant. These curves are generated for a hyperbolic tangent mean streamwise velocity profile with spreading rates estimated from DNS data with a laminar boundary layer upstream. ${ }^{11}$ These inviscid stability calculations accounted for the finite depth of the cavity by forcing the eigenfunctions to have zero normal velocity there. A range of $D / \theta$ that is typical of DNS conditions is shown, and it is clear that the finite depth has a large impact on the growth rate when $D / \theta$ is less than about 20 . The plot also shows why certain conditions lead to multiple modes with similar amplitudes, while other conditions lead to a single dominant mode. These curves were found to well predict the dominant mode in the DNS calculations, as well as under which flow conditions multiple nearly equal modes would be found. Predictions for the linear stability eigenfunctions are compared to spectra from the DNS below in figure 4. The calculations well capture the shape of the modes, except in the impingement region very near the trailing edge. Cattafesta et al. ${ }^{7}$ also showed good agreement for the initial exponential growth rate (close to the leading edge) with linear stability theory. The phase speed of the disturbances is less sensitive to various parameters such as $M$ and $L / \theta$ and $D / \theta$, but nevertheless variations based on linear stability analysis are sufficient to explain some of the scatter in the frequencies of oscillation from experiments, as noted above.

In analyzing the behavior of the shear layer, most investigators have implicitly assumed that the shear layer behavior can be described in isolation, i.e. as if it were a free shear layer. Nonparallel effects, and the coupling of the flow inside the cavity are of course neglected in such an analysis. An alternative analysis of the global instability modes, which requires spatial DNS as input, is being developed for the cavity by Theofilis. ${ }^{61}$ Such an analysis will in future certainly help shed light on the 


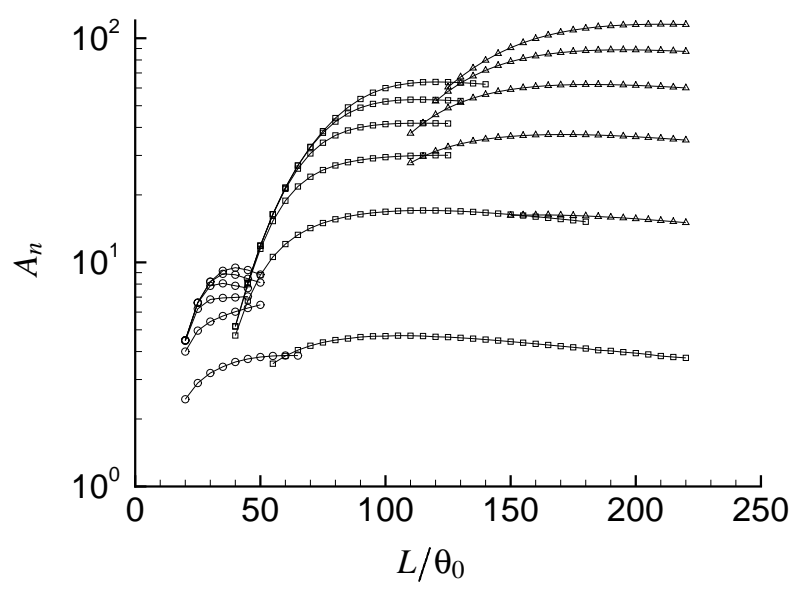

Figure 1: The inviscid shear layer amplification, $A_{n}$ (arbitrary scale) versus $L / \theta_{0}$ for different $D / \theta_{0}$, from ref. ${ }^{11}$, for $M=0.2$. The dominant mode is shown at each $L / \theta_{0}$, with $n=1$ (०), $n=2$ (口), and $n=3(\triangle)$ ). The curves for different $D / \theta_{0}$ are, for each mode, monotonically increasing from bottom to top with $D / \theta_{0}=$ $5,10,15,20,25$, and 30 .

bifurcations from steady to oscillating flow, and has the additional advantage of being applicable as well to flows that are three-dimensional in the mean.

Of course, the shear layer amplification and phase speed of the waves are only one factor that determines the overall frequency and amplitude of the resonant modes. The sound generation and receptivity processes at the edges must also be modeled to yield quantitative predictions. The linear analysis of Tam and Block ${ }^{60}$ idealizes the sound generation at the downstream edge as a simple line source along the trailing edge that produces a compression during that part of the cycle when the shear layer is deflected into the cavity, thus creating an impingement zone and a transient region of high pressure. The arguments presented are based on the Schlieren visualizations of Krishnamurty, ${ }^{31}$ and the observation that the pressure fluctuations near the edge are in phase inside and atop the cavity. Recent results from DNS ${ }^{11}$ examined the behavior of the density fluctuations in a region near the source and in the far field, and seemed to confirm the scaling implied by Tam's model. Predictions for the relative overall amplitude based on a combined shear layer instability and sound generation model where, in turn, able to effectively predict the onset of Rossiter mode oscillations and transition to wake mode oscillations (discussed in section 4.4) in the DNS results. ${ }^{11}$

Weiner-Hopf techniques were used by Crighton to model frequency selection in edge-tones, ${ }^{14}$ and have recently been applied by Kerschen ${ }^{5,26}$ to the supersonic cavity. This latter technique solves, simultaneously, the interaction of waves with the shear and the receptivity problem at the leading edge, at least for the case of a zero-thickness shear layer.

Cain et al. ${ }^{5}$ have developed a computer design tool, Cavity Acoustics Modeling Software (CAMS), that models the various components in the feedback loop, including finite thickness shear layer instability calculations, and a empirical equation to determine the shear layer spreading as a function of the amplification of the disturbances and a level of background turbulence. The computation is iterative, proceeding around the loop until the amplitude prediction converges. They have developed a similar model to predict jet screech, with a vortex-shock interaction model replacing sound generation at the cavity edge. ${ }^{4}$ Both models are design to run in a few seconds on a desktop computer. Williams and Fabris report good agreement between the CAMS predictions and their experimental results. ${ }^{64}$ It would be of interest to obtain further validation of this tool by comparing results with more of the recent experiments. These analytical and semi-analytical models for the individual components in the feedback loop may ultimately prove useful in the development of model-based control schemes. Further work is needed, however, to incorporate the effects of actuation, and to cast the model in a control-theoretic framework. ${ }^{44}$

Two areas that have received comparatively little attention are nonlinearities and three-dimensionality. There is evidence to suggest that under some conditions, there can be strong interactions between the modes of oscillation. Cattafesta et al. ${ }^{7}$ have observed mode switching, where the cavity periodically switches between two different Rossiter frequencies. They used a variety of tools (Short Time Fourier Transform, wavelets, and higher-order spectral techniques) to analyze signals from subsonic cavity resonance. They hypothesize that nonlinear interactions amongst the Rossiter modes produce low frequency modulation of the oscillations. For example, when three Rossiter modes were present and satisfied a criterion: $S t_{3}-\left(S t_{1}+S t_{2}\right) \approx 0$ the most significant low frequency modulation was present. Williams and Fabris ${ }^{65}$ have noted that enhancing a particular mode leads to reductions in amplitude of neighboring modes and, conversely, suppressing a single mode can lead to amplification of neighboring modes. This suggests a competition amongst modes, and could be associated with relative changes to the spreading of the shear layer as the amplitudes of the various modes are adjusted.

Analysis and most computations (see next section) have modeled cavity resonance as strictly twodimensional. Indeed many of the observations seem to 
confirm the validity of this assumption in describing at least certain aspects of cavity resonance. However, consideration of three-dimensional geometry and boundary layer turbulence may be important as models of cavity resonance become more sophisticated. For example, some of the actuators that have been used in control experiments introduce significantly three-dimensional disturbances (such as "sawtooth" spoilers, or the fluidic actuators of ref. ${ }^{39}$ ). Future challenges include understanding the detailed mechanism by which these devices interfere with the resonant modes.

\section{Numerical Simulations}

\subsection{Turbulence Modeling}

Two dimensional simulations employing unsteady RANS turbulence models have been used by a number of investigators in the subsonic, ${ }^{3,17,24,53}$ transsonic and supersonic, ${ }^{23,24,32,42,54,55,67,68}$ and hypersonic ${ }^{8}$ regimes. Most of the calculations have concentrated on rectangular cavities, with $L / D$ ranging from about 2 to 10 . The earlier works utilized zero equation turbulence models, and later works generally used two-equation models in the $k-\varepsilon$ or $k-\omega$ framework. Experimental validation of these simulations include good agreement with frequencies of oscillation from experiments (and as predicted by Rossiter's formula), and in some cases reasonable agreement for mean velocity profiles, RMS pressure fluctuations along the cavity walls, and the peak amplitudes in pressure spectra.

Given the dominance of large scale vortices in the instability process, the flow would at seem a good candidate for Large Eddy Simulation. Complicating this, however, is the recognition that proper resolution of the large scale turbulence in the incoming boundary layer flow will require very large grids, for even moderate Reynolds number cavities. One alternative, which has been used in practice, is to use RANS in the boundary layer upstream of the cavity, switching to LES over the cavity. Shieh and Morris ${ }^{53}$ have computed subsonic cavities with such a hybrid RANS/LES methodology, but only in two spatial dimensions. Supersonic results have been published ${ }^{55}$ for three-dimensional LES (Smagorinsky) of a rectangular cavity with $L / D=4.5, L / H=4.5$, $M=2$, and $R e_{L}=4.5 \times 10^{6}$, and for the same cavity with $M=1.5$ by the Lockheed group. ${ }^{57}$ In both cases the run conditions correspond to the high-speed data of Shaw. ${ }^{50}$ The Lockheed study also compared results with an Euler method (which relies on numerical viscosity as a crude turbulence model). Peak amplitudes in the pressure spectra showed reasonable agreement with the experiments, but were sensitive to numerical diffusion and modeling assumptions and grid densities. ${ }^{57}$ Sinha et $a l .{ }^{55}$ report vorticity iso-surfaces and Reynolds stresses at the cavity mid-length and mid-span that show very little three-dimensionality, but it is not clear whether threedimensionality would evolve correctly in the absence of forcing from the upstream boundary layer.

Generally speaking, these simulations show promise, but cannot yet be regarded as complete enough to validate in detail the efficacy of compressible turbulence models for these separated, oscillating flows. Indeed, the lack of any detailed mean flow or turbulence statistics measurements (and the difficulty of obtaining such in the high-speed flows) further hampers the effort. Key issues regarding three-dimensionality of the large-scale oscillations also remain to be addressed, as discussed in the previous section.

\subsection{Sound Radiation}

As discussed in section 3, the efficiency of the sound generation process at the downstream edge is a factor in determining the overall amplitude of the oscillations, and therefore also the mixture of modes that will be present for a given geometry and flow conditions, and the possible nonlinear interaction of modes. Accurate simulation of the radiated acoustic field together with the unsteady hyrdodynamic field is computationally challenging due to the relatively small amplitude of the acoustic field (compared to large scale turbulence), and due to the long distances over which the waves must propagate with little viscous attenuation. Work over the past 10 years (see, for example, the review by Lele $^{35}$ ) in Computational Aeroacoustics (CAA) has drawn attention to various computational artifacts that can lead to both poor resolution of the acoustic radiation and spurious flow oscillations. The former is the result of excessive dispersion and dissipation that can result from low-order and upwind finite difference and finite volume discretizations (and indeed from insufficient grid resolution), and the latter can result from excessive reflection of disturbances from artificial boundary conditions in the free stream.

It has been found in numerous studies that compact high-order finite-difference schemes ${ }^{34}$ and optimized compact and explicit finite-difference schemes (such as dispersion-relation-preserving (DRP) schemes $^{59}$ ), together with high-order-accurate Runge-Kutta schemes for time advancement, can minimize errors due to dispersion and dissipation of acoustic waves. For example, compact finite-difference schemes were used in DNS studies of mixing layers, ${ }^{13}$ jets $^{15,16,36}$ and the cavity ${ }^{10}$ that have included a detailed assessment of grid convergence, the effect of boundary conditions, and validation of the acoustic fields by comparison with acoustic analogy solutions computed with source terms determine by 
the DNS. Cavity computations utilizing the DRP scheme by Shieh and Morris ${ }^{53}$ also capture the acoustic radiation directly. It should be noted that these methods are usually more efficient than lower-order methods, as they require many fewer nodes to resolve a given feature of the flow. While high-order methods are hard to adapt to very complex geometry, they may easily be adapted to the block Cartesian structure of the cavity geometry, ${ }^{10,53}$ and work continues to adapt these methods for generalized coordinate systems.

Boundary conditions also present a technical challenge in aeroacoustic computations. Artificial boundaries (inflow/outflow/normal) must allow vortical and acoustic waves to pass freely with minimal reflection. Indeed, the development of accurate boundary conditions has been a pacing item in the development of CAA in general. Previous work has shown the repeated reflections of these waves can give rise to self-forcing of the flow, in a process that can be difficult to distinguishable from physical instability. ${ }^{12}$ For resonant flows such as the cavity, it is therefore important to document by numerical experimentation that the instabilities are indeed independent of the location of artificial boundaries.

\subsection{Direct Numerical Simulations}

DNS has the advantage of directly resolving all the scales of motion (as well as the radiated acoustic field as discussed below) without recourse to turbulence models, but for the cavity flow is presently limited to low Reynolds number where the upstream boundary layer is laminar or transistional. In recent work at Caltech, ${ }^{10,11}$ we have performed a series of two-dimensional DNS calculations over a wide range of parameters $2<L / D<8$, $30<R e_{\theta}<80,20<L / \theta<120$ and $0.2<M<0.8$, with accurate resolution of the resulting acoustic radiation as discussed in the last section. Further details of the code and its validation is given in the references. Experimental verification of the results is somewhat limited due to low Reynolds number considered. The early laminar boundary layer experiments of Kirshnamurty are closest to the DNS data, and a qualitative comparison of Schlieren images for the cavity and its radiated acoustic field (Figure 2) shows a remarkably similar change in structure of the acoustic field as the Mach number is varied.

\subsection{Wake Mode}

Gharib and Roshko ${ }^{20}$ observed in their incompressible experiments for an axisymmetric cavity that as the length of the cavity (relative to the upstream boundary layer thickness) was increased, there was a substantial change in the behavior of the cavity oscillations. Under these conditions, the flow was characterized by a large scale (dimensions of the cavity depth) shedding from the cavity leading edge. In two-dimensional DNS, Colonius et al. ${ }^{10,11}$ have observed a very similar transition. In both experiment and computation, the boundary layer upstream of the cavity was laminar. The wake mode transition has also been observed in two-dimensional RANS calculations at higher Reynolds numbers. ${ }^{3,17,53}$
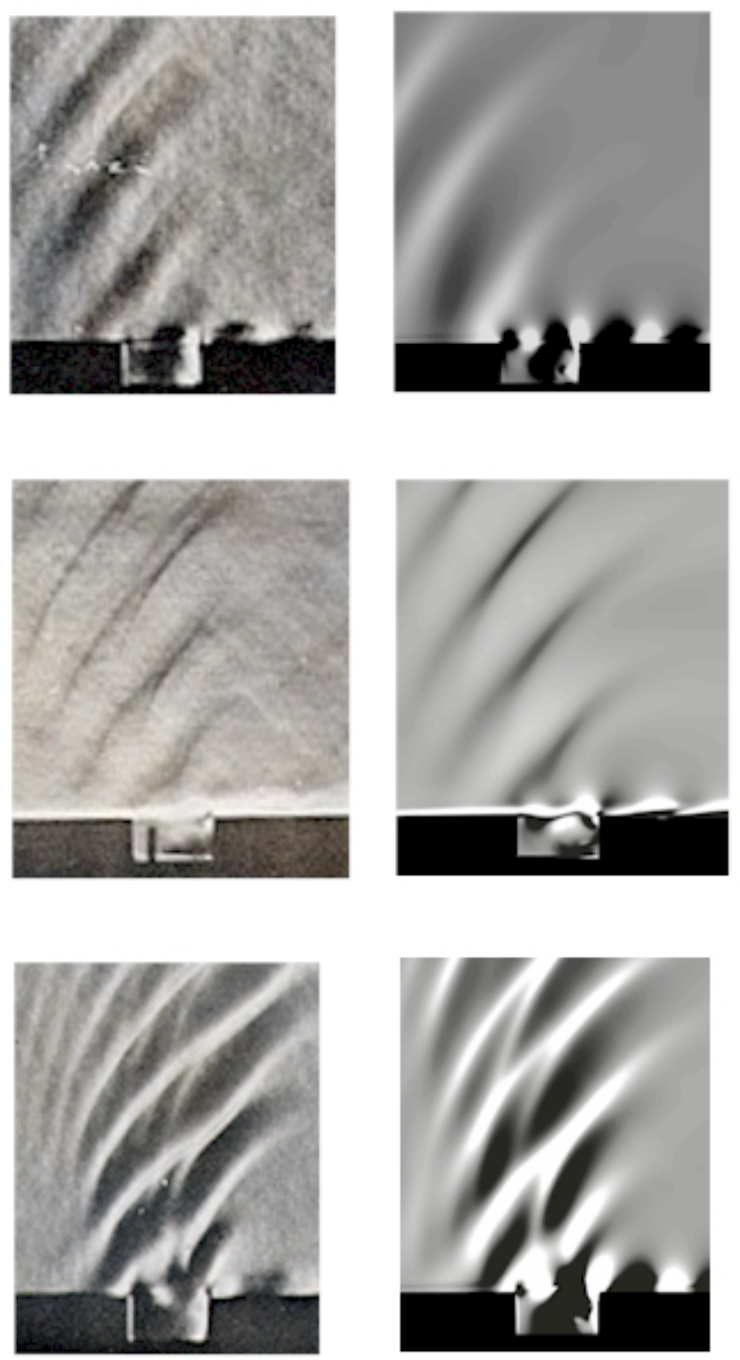

Figure 2: Comparison of Schlieren Photographs ${ }^{31}$ with contours of density gradient from the DNS. ${ }^{11}$ Left column are the experimental results at $M=0.640 .7$, and 0.8 (top to bottom) and the right column are density gradient contours from DNS at similar Mach numbers. Note that middle row have the knife-edge horizontal while the top and bottom have knife-edge vertical. 
Figure 3 shows snapshots of the vorticity field in wake mode over one period of oscillation. Flow visualizations from wake mode also show striking similarity to oscillations seen in gas flows in pipes with closed side branches ${ }^{30}$ (with turbulent boundary layers upstream of the branch). The shed vortex has dimensions of nearly the cavity size, and as it is forming, irrotational free stream fluid is directed into the cavity, impinging on the cavity base. The vortex is shed from the leading edge and ejected from the cavity in a violent event.
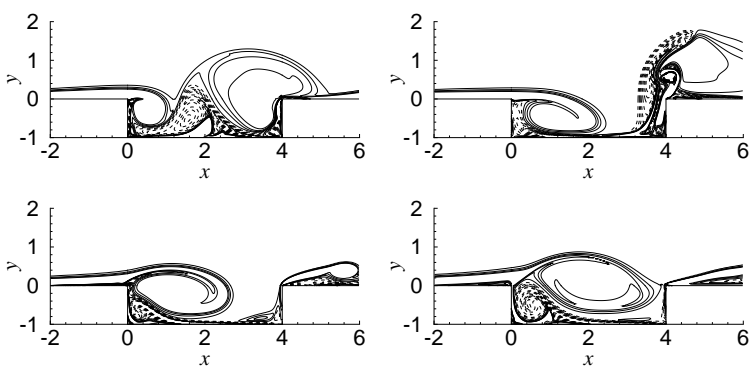

Figure 3: Instantaneous vorticity contours from ${ }^{11}$ showing a $M=0.6, L / D=4, R_{e}=59, L / \theta=102$ cavity in wake mode oscillation. Only a small portion of the computational domain near the cavity is shown.

The mean flow within the cavity is significantly different in wake mode. The mean streamlines in wake mode are significantly deflected above the cavity, and there is an impingement of the freestream flow on the rear wall, with secondary separation downstream of the cavity. The terminology "closed" and "open" cavities is used to denote the situation where the flow does or does not reattach to the floor of the cavity, respectively. Open cavities are thought to exist for $L / D<13$. The mean flow in wake mode in the DNS exhibited no mean or instantaneous reattachment to the cavity bottom. Flow visualizations indicate as well that there is no instantaneous reattachment of the primary shear layer on the cavity bottom, though there is significant deflection of freestream fluid into the cavity during portions of the cycle. This greatly increases drag on the cavity, with increases from Rossiter-type oscillations by about a factor of 10 (similar increases were reported in $\operatorname{ref}^{20}$ ).

Colonius et al. ${ }^{11}$ have performed an extensive mapping of the transition to wake mode in parameter space. A key point is that the frequency of oscillation in wake mode is to be nearly constant with Mach number (from 0.4 to 0.8 ) and it would appear that the instability is in this regime is purely hydrodynamic in nature. Based on similarities of the mean flow profiles with those of wakes and shear layers between streams of opposing direction, it was speculated that the transition may be the result of an absolute instability. A remarkably consistent prediction for the transition was derived based on linear stability of the shear layer as it exists in Rossiter-type oscillations. The hypothesis is that the transition occurs as the shear layer instabilities grow to very large values, either due to the length of the cavity, or similar parametric changes that lead to larger amplitude oscillations.

It is important to note that at similar values of $M$ and $L / D$, but at higher Reynolds number, and with turbulent upstream boundary layers, wake mode has not been observed in cavity experiments. It is known that cavity resonance is stronger for laminar flow than turbulent flow, ${ }^{31}$ apparently because the additional turbulent spreading of the shear layer leads to overall lower levels of amplification. This may be what prevents transition to wake mode at higher Reynolds numbers. Similarly, the turbulent flow within the cavity is very likely to be much less organized in three-dimensional flow. Smaller recirculation, especially near the upstream edge, may also deter the transition. Neverthless, it may be important to help insure that aircraft designs avoid this regime due to the greatly enhanced drag and strong flow within the cavity.

\section{LOW-ORDER DYNAMICAL SYSTEM APPROACH TO MODELING}

Cavity oscillations exhibit several phenomena characteristic of a low-dimensional dynamical system. For instance, for a fixed flow velocity and momentum thickness there is a minimum cavity length below which oscillations do not occur, as seen in low Mach-number experiments by Sarohia. ${ }^{46}$ Similarly, there is a minimum velocity and momentum thickness above which oscillations do not occur (when other parameters are held constant). When the length is gradually increased, the frequency of oscillation decreases linearly, but once a critical value of length is reached, the frequency jumps, as the cavity switches to a higher Rossiter mode. Mode switching has been observed. One curious observation is that hysteresis, which occurs in mode selection in edge tones, has never been observed in cavity oscillations.

Many of these qualitative similarities suggest the possibility of describing the cavity flow with a low-order model. The goal is to understand the various transitions more rigorously, as bifurcations in a dynamical system, and ultimately to control the cavity oscillations with a model-based feedback law. One route to a nonlinear low-order system model is through the Proper Orthogonal Decomposition and Galerkin projection of the POD modes onto the governing equations. The Proper Orthogonal Decomposition (POD) is a commonly used tool for extracting coherent structures from data, either experimental or computational. ${ }^{25,56}$ This approach, with 
varying success, has now been employed in a variety of different flows. We briefly discuss here the theoretical framework and some results specifically related to cavity flows.

Given a set of data, represented as a function of space and time, the POD determines a basis set of orthogonal functions of space which span the data optimally in the $L^{2}$ sense. For data that has been discretized in space and time, the POD modes are most easily computed using the method of snapshots. If $N$ "snapshots" are given one constructs the expansion:

$$
u(\mathbf{x}, t)=\sum_{j=1}^{N} a_{j}(t) \varphi_{j}(\mathbf{x})
$$

where $\mathbf{x}$ is the vector of discretized spatial points. The functions $\varphi_{j}$ are called the POD modes (also called Karhunen-Loève eigenfunctions, or empirical eigenfunctions), and are computed from the discrete data by efficient algorithms for singular value decomposition.

POD is a useful decomposition of a flow field when the coefficients, $a_{j}$, decrease rapidly for large $j$. This indicates the percentage of "energy" that is captured by the first few modes is significant. Several investigators have computed POD modes for cavity oscillations based on numerical simulations. $^{3,44,55,62}$ They have all found that a significant fraction of the total energy is captured in just a few modes. For cavities where one mode is clearly dominant, Rowley and Colonius ${ }^{44}$ find that $70-80 \%$ of the disturbance energy is captured in just 2 modes. These two modes capture two views of the single resonant mode with a phase shift of $180^{\circ}$. They found that for a cavity with two significant resonant peaks, the first 4 POD modes captured about $50 \%$ of the energy (again, the 4 POD modes account for the two resonant modes at 180 degree phase shift). The lower fraction of total energy captured by the resonant modes seems to suggest that nonlinear interactions between modes leads to more complicated dynamics. Contours of the normal velocity fluctuations from the first 4 modes are reproduced in Figure 4, where they are compared to linear stability calculations (describe in section 3) and to the Discrete-Fourier Transform (in time) of the DNS data from which they were generated. All three present a nearly identical picture of the two resonant modes.

By projecting the governing Partial Differential Equations onto the POD modes, and truncating the resulting system of equations to low-order, a (nonlinear) system of Ordinary Differential Equations is obtained. For example, suppose the governing equation are written, symbolically, as:

$$
\partial_{t} u=D_{\lambda}(u)
$$
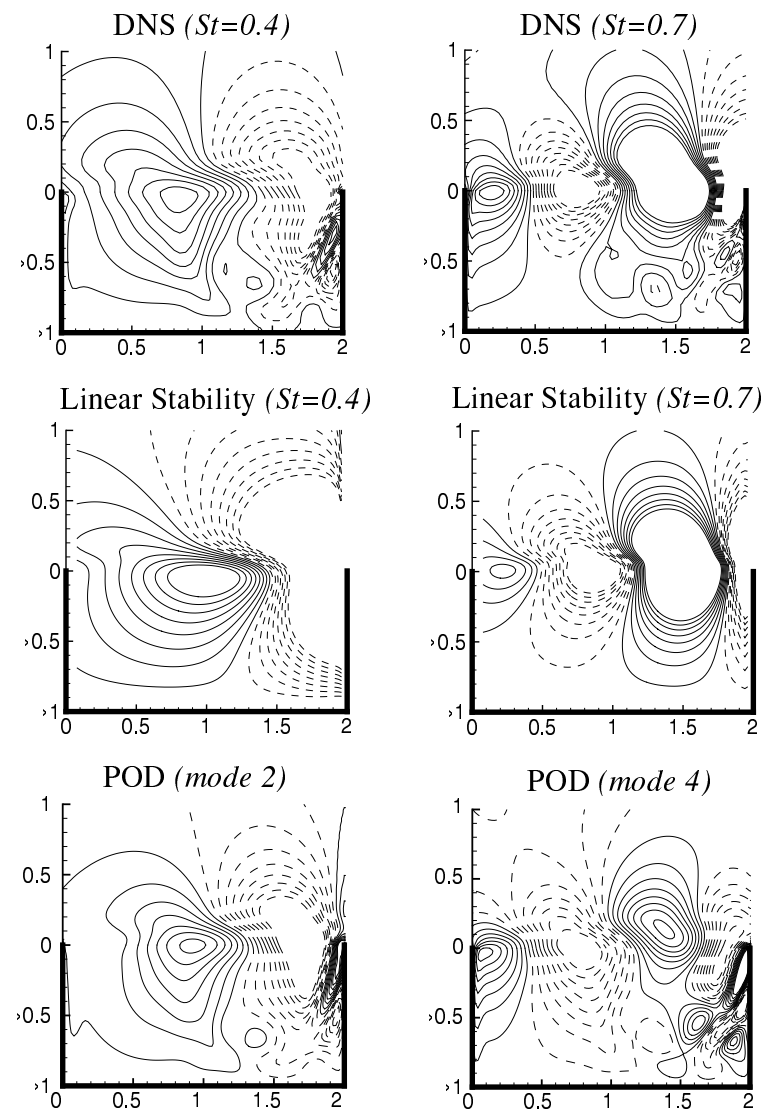

Figure 4: Comparison of the Discrete Fourier transform in time of the DNS results at $S t=0.4$ and $S t=0.7$ (real parts), eigenfunctions from quasi-parallel stability analysis, and 2 modes from the Proper Orthogonal Decomposition. $M=0.6, L / D=2, \operatorname{Re}_{\theta}=59, L / \theta=51$. Only a small portion of the computational domain near the cavity is shown. Data from. ${ }^{44}$

where $u(x, t)$ is a vector of the dependent variables, a function of space and time, and $D_{\lambda}$ is a nonlinear spatial differential operator which depends on some parameters $\lambda$ (for instance $\lambda=(M, L / \theta, R e)$ ). Substituting the POD expansion above, and taking an inner product with $\varphi_{k}$ gives

$$
\dot{a}_{k}=\left\langle D_{\lambda}(u), \varphi_{k}\right\rangle, \quad k=1, \ldots, n,
$$

where $\langle\cdot, \cdot\rangle$ denotes the $L^{2}$ inner product. Since $u$ is written in terms of the time coefficients, this yields a set of ODE:

$$
\dot{a}=f(a, \lambda),
$$

where $a=\left(a_{1}, \ldots, a_{n}\right)$. Whether the resulting equations are an appropriate model of cavity resonance depends 
on how well the reduced-order system tracks the original system, as a function of time. In preliminary work, Rowley and Colonius ${ }^{44}$ experimented with systems of 2 to 20 modes and found that the models performed very well for short times (two or three periods of oscillation), but then began to deviate. There was little advantage to retaining more modes, and, in fact, the system that retained only those POD modes corresponding to the resonant modes performed the best. Unfortunately, the model ultimately did not predict the correct amplitude of the resulting limit cycles, but it was possible that an insufficient number of snapshots were used to generate the POD modes such that the saturation process could not be captured. Further work is needed to rectify this issue but we note that in a controller model, it may be sufficient to predict the system behavior for relatively short times.

Rowley and Colonius ${ }^{44}$ have noted that it is desirable to scale the governing equations in such a way that as many of the governing parameters, as is possible, appear explicitly in the equations of motion. These parameters will then be represented in the low-order system of equations. This idea of rescaling the equations is a special case of a more general idea of mapping different domains (geometries) into a canonical geometry. ${ }^{28}$ This has been used successfully for unsteady flow through diffusers with varying diffuser angle. ${ }^{28}$ If a sufficiently broad selection of snapshots (i.e. from simulations with different geometry) are used to "train" the POD modes, behavior of the system with varying parameters may be possible. For the cavity, it is of course not possible to rescale the equations such that both $L / \theta$ and $L / D$ appear as a parameter. However, Rowley and Colonius ${ }^{44}$ have found that the POD modes where much more strongly dependent on $L / \theta$ (which is consistent with ideas from linear stability), than with $L / D$, provided that $D / \theta$ was large enough.

The POD/Galerkin approach seems valuable as a systematic way to derive low order cavity resonance models, but more work is needed before these are of practical value in developing feedback control strategies. The most significant issue that remains to be addressed is whether the effects of flow actuation (and the dynamics of the actuated flows) can be appropriately captured in the low-order system. One approach, used by Gillies ${ }^{21}$ for the circular cylinder wake, is to use snapshots from the flow under a variety of levels of external excitations, in an attempt to capture the appropriate dynamics.

\section{SUMmARY AND FUtURE TRENDS}

Flow control experiments have been, for the most part, successful at reducing the amplitude of tones, and sometimes broadband noise, in flows over open cavities over a range of Mach numbers from nearly incompressible to moderately supersonic. Recent work $^{58}$ on highfrequency forcing of the shear layer, in particular using so called power resonance tubes and a simple (passive) rod in crossflow upstream of the cavity, showed remarkable reductions of both tone amplitudes and broadband noise, and the investigators suggest that this may be sufficient for practical application to full scale weapons bay problems, provided that the actuation mechanisms are found to scale appropriately.

Aside from the high frequency forcing, which is thought to primarily enhance the rate at which the energy cascade deprives energy from the large scales, there is no clear evidence of the precise mechanism for attenuation of a particular mode (or multiple modes) in the lower frequency results. If model based closed-loop control is to be implemented, further progress in correctly predicting the amplitudes of tones will be required. One promising approach for such a model is linear modeling of the shear layer instability, sound generation, and receptivity problems as outlined above, together with a nonlinear extension that describes how the modes impact the spreading of the shear layer. More recent work on distilling low order models based on the Proper Orthogonal Decomposition, and the projection of the governing equations onto the POD modes, also appears to show promise, though further work will be required to allow such models to capture the dynamics of both the natural and controlled cavities.

Some issues that have hitherto received very little attention are nonlinear interactions of modes (for which there is experimental evidence), and threedimensionality, both with respect to the natural cavity instabilities and especially for actuators that introduce three-dimensional disturbances. Numerical simulations are likely to play a key role in such investigations. Simulation efforts should be guided by recent advances in the area of computational aeroacoustics in order to avoid significant artificial dispersion and dissipation of the acoustic component of the feedback loop. Direct numerical simulations offer a glimpse into the details of nonlinear cavity dynamics, but are restricted to very low Reynolds numbers at present time. Large Eddy Simulations at moderate Reynolds numbers show promise in bringing accurate computation at conditions that are more realistic of the relevant applications. It would appear that these simulations will also necessitate more detailed experimental data, especially turbulence statistics, in order to be carefully validated. 


\section{ACKNOWLEDGEMENTS}

I am grateful to my collaborators at Caltech, Mr. Clancy Rowley, Dr. Amit Basu,and Prof. Richard Murray, for their contributions to the cavity flow project. Thanks are due to Clancy as well for reading a draft of this report. I have also benefited from numerous discussions on cavity acoustics with A. Cain,M. Gharib, A. Roshko, D. Williams. The support by AFOSR under grant F4962098-1-0095 with technical monitor Dr. Thomas Beutner is gratefully acknowledged.

\section{REFERENCES}

[1] T. R. Bewley. Flow control: New challenges for a new renaissance. Progress in Aerospace Sciences, to appear, 2001.

[2] W. K. Blake and A. Powell. The development of contemporary views of flow-tone generation. In Recent Advances in Aeroacoustics, pages 247-345. Springer-Verlag, 1986.

[3] A. Cain, R. A. D., D. M. Bortz, H. T. Banks, and R. C. Smith. Optimizing control of open bay acoustics. AIAA Paper 2000-1928, 2000.

[4] A. B. Cain and W. W. Bower. Modeling supersonic jet screech: Diffferential entrainment and amplitude effects. AIAA Paper 96-0916, 1996.

[5] A. B. Cain, W. W. Bower, F. McCotter, and W. W. Romer. Modeling and prediction of weapons bay acoustic amplitude and frequency. Technical report, VEDA Inc., 1996.

[6] L. N. Cattafesta III, S. Garg, M. Choudhari, and F. Li. Active control of flow-induced cavity resonance. AIAA Paper 97-1804, June 1997.

[7] L. N. Cattafesta III, S. Garg, M. S. Kegerise, and G. S. Jones. Experiments on compressible flowinduced cavity oscillations. AIAA Paper 98-2912, June 1998.

[8] N. Chokani. Prediction of hypersonic cavity flow dynamics. AIAA Paper 99-0171, 1999.

[9] N. Chokani and I. Kim. Supression of pressure oscillations in an open cavity by passive pneumatic control. AIAA Paper 91-1729, 1991.

[10] T. Colonius, A. J. Basu, and C. W. Rowley. Numerical investigation of the flow past a cavity. AIAA Paper 99-1912, May 1999.
[11] T. Colonius, A. J. Basu, and C. W. Rowley. On self-sustained oscillations in two-dimensional compressible flow over rectangular cavities. Journal of Fluid Mechanics, submitted, 2001.

[12] T. Colonius, S. K. Lele, and P. Moin. Boundary conditions for direct computation of aerodynamic sound. AIAA Journal, 31(9):1574-1582, 1993.

[13] T. Colonius, S. K. Lele, and P. Moin. Sound generation in a mixing layer. Journal of Fluid Mechanics, 330:375-409, 1997.

[14] D. G. Crighton. The jet edge-tone feedback cycle; linear theory for the operating stages. Journal of Fluid Mechanics, 234:361-391, 1992.

[15] J. Freund, S. Lele, and P. Moin. Numerical simulation of a Mach 1.92 turbulent jet and its sound field. AIAA Journal, 38(11):2023-2031, 2000.

[16] J. B. Freund. Acoustic sources in a turbulent jeta direct numerical simulation study. AIAA Paper 99-1858, 1999.

[17] D. Fuglsang and A. Cain. Evaluation of shear layer cavity resonance mechanisms by numerical simulation. AIAA Paper 92-0555, 1992.

[18] M. Gad-el-Hak. Modern developments in flow control. Applied Mechanics Reviews, 49(7):365-379, 1996.

[19] M. Gharib. Response of the cavity shear layer oscillations to external forcing. AIAA Journal, 25(1), Jan. 1987.

[20] M. Gharib and A. Roshko. The effect of flow oscillations on cavity drag. Journal of Fluid Mechanics, 177:501-530, 1987.

[21] E. Gillies. Low-dimensional control of the circular cylinder wake. Journal of Fluid Mechanics, 371:157-178, 1998.

[22] J. E. Grove and R. M. Birkbeck. Acoustic and separation characteristics with bay leading edge blowing. AIAA Paper 2000-1904, 2000.

[23] W. L. Hankey and J. S. Shang. Analysis of pressure oscillation in an open cavity. AIAA Journal, 18(8):892-898, 1980.

[24] J. Henderson, K. Badcock, and B. E. Richards. Subsonic and transonic transitional cavity flows. AIAA Paper 2000-1966, 2000. 
[25] P. Holmes, J. L. Lumley, and G. Berkooz. Turbulence, Coherent Structures, Dynamical Systems and Symmetry. Cambridge University Press, 1996.

[26] E. J. Kerschen. Sound generated at the downstream end of a cavity under a supersonic stream. Bulletin of the American Physical Society, 45(9):138, 2000.

[27] T. Kestens and F. Nicoud. Active control of an unsteady flow over a rectangular cavity. AIAA Paper 98-2348, 1998.

[28] I. Kevrekidis and P. Holmes. Personal communication.

[29] S. Kikuchi and Y. Fukunishi. Active flow control technique using piezo-film actuators applied to the sound generation by a cavity. ASME FEDSM99$7232,1999$.

[30] P. C. Kriesels, M. C. A. M. Peters, A. Hirshberg, A. P. J. Wijnands, A. Iafrati, G. Riccardi, R. Piva, and J. C. Bruggeman. High aplitude vortex-induced pulsations in a gas transport system. Journal of Sound and Vibration, 184(2):343-368, 1995.

[31] K. Krishnamurty. Sound Radiation from Surface Cutouts in High Speed Flow. PhD thesis, California Institute of Technology, 1956.

[32] A. Lamp and N. Chokani. Computation of cavity flows with supression using jet blowing. Journal of Aircraft, 34(4):545-551, 1997.

[33] A. Lamp and N. Chokani. Control of cavity resonance using steady and oscillatory blowing. AIAA Paper 99-0999, 1999.

[34] S. K. Lele. Compact finite difference schemes with spectral-like resolution. Journal of Computational Physics, 103(1):16-42, 1992.

[35] S. K. Lele. Computational aeroacoustics: A review. AIAA Paper 97-0018, 1997.

[36] B. Mitchell, S. Lele, and P. Moin. Direct computation of the sound generated by vortex pairing in an axisymmetric jet. Journal of Fluid Mechanics, 383:113-142, 1999.

[37] M. A. Pinney and J. Leugers. Experimental investigation of the impact of internal/external weapons carriage on a generic aircraft configuratoin. Technical Report WL-TR-96-3110, Wright Laboratories, 1996.

[38] A. Powell. On the edgetone. Journal of the Acoustical Society of America, 33:395, 1961.
[39] G. Raman, S. Raghu, and T. J. Bencic. Cavity resonance supression using miniature fluidic oscillators. AIAA Paper 99-1900, 1999.

[40] D. Rockwell and E. Naudascher. Review-selfsustaining oscillations of flow past cavities. Transactions of the ASME, Journal of Fluids Engineering, 100, June 1978.

[41] D. Rockwell and E. Naudascher. Self-sustaining oscillations of impinging free shear layers. Annual Review of Fluid Mechanics, 11:67-94, 1979.

[42] A. Rona and W. Dieudonne. A flow-resonant model of transonic laminar open cavity instability. AIAA Paper 2000-1967, 2000.

[43] J. E. Rossiter. Wind-tunnel experiments on the flow over rectangular cavities at subsonic and transonic speeds. Aeronautical Research Council Reports and Memoranda, No. 3438, Oct. 1964.

[44] C. W. Rowley, T. Colonius, and R. M. Murray. Pod based models of self-sustained oscillations in the flow past an open cavity. AIAA Paper 2000-1969, 2000.

[45] R. L. Sarno and M. E. Franke. Suppression of flowinduced pressure oscillations in cavities. Journal of Aircraft, 31(1):90-96, 1994.

[46] V. Sarohia. Experimental and Analytical Investigation of Oscillations in Flows Over Cavities. $\mathrm{PhD}$ thesis, California Institute of Technology, 1975.

[47] V. Sarohia. Experimental investigation of oscillations in flows over shallow cavities. AIAA Journal, 15:984-991, July 1977.

[48] V. Sarohia and P. F. Massier. Control of cavity noise. Journal of Aircraft, 14(9):833-837, 1977.

[49] L. Shaw. Active control for cavity acoustics. AIAA Paper 98-2347, 1998.

[50] L. Shaw. High speed application of active flow control for cavity acoustics. AIAA Paper 2000-1926, 2000 .

[51] L. Shaw and L. McGrath. Active control of shallow cavity acoustic resonance. AIAA Paper 96-1949, 1996.

[52] L. Shaw and S. Northcraft. Closed loop active control for cavity acoustics. AIAA Paper 99-1902, May 1999.

11 
[53] C. M. Shieh and P. J. Morris. Parallel computational aeroacoustic simulation of turbulent subsonic cavity flow. AIAA Paper 2000-1914, 2000.

[54] S. H. Shih, A. Hamed, and J. J. Yeuan. Unsteady supersonic cavity flow simulations using coupled $k-\varepsilon$ and Navier-Stokes equations. AIAA Journal, 32(10):2015-2021, 1994.

[55] N. Sinha, S. Arunajatesan, and L. S. Ukeiley. High fidelity simulation of weapons bay aeroacoustics and active flow control. AIAA Paper 2000-1968, 2000 .

[56] L. Sirovich. Turbulence and the dynamics of coherent structures, parts I-III. Quarterly of Applied Mathematics, XLV(3):561-590, Oct. 1987.

[57] B. R. Smith, J. R. Jordan, E. E. Bender, S. N. Rizk, and L. L. Shaw. Computational simulation of active control of cavity acoustics. AIAA Paper 200-1927, 2000.

[58] M. J. Stanek, G. Raman, V. Kibens, J. A. Ross, J. Odedra, and J. W. Peto. Control of cavity rsonance through very high frequency forcing. AIAA Paper 2000-1905, 2000.

[59] C. Tam and J. Webb. Dispersion-relationpreserving finite-difference schemes for computational acoustics. Journal of Computational Physics, 107(2):262-281, 1993.

[60] C. K. W. Tam and P. J. W. Block. On the tones and pressure oscillations induced by flow over rectangular cavities. Journal of Fluid Mechanics, 89(2):373-399, 1978.

[61] V. Theofilis. Globally unstable basic flows in open cavities. AIAA Paper 2000-1965, 2000.

[62] L. Ukeiley, J. Seiner, N. Sinha, and S. Arunajatesan. Low-dimensional description of cavity flows. Bulletin of the American Physical Society, 45(9): 138, 2000.

[63] A. D. Vakili and C. Gauthier. Control of cavity flow by upstream mass-injection. Journal of Aircraft, 31(1):169-180, 1994.

[64] D. R. Williams and D. Fabris. Closed-loop control in cavities with unsteady bleed forcing. AIAA Paper 2000-0470, 2000.

[65] D. R. Williams and D. Fabris. Experiments on controlling multiple acoustic modes in cavities. AIAA Paper 2000-1903, 2000.
[66] J. M. Wiltse and A. Glezer. Direct excitation of small-scale motions in free shear flows. Physics of Fluids, 10(8):2026-2036, 1998.

[67] X. Zhang and J. A. Edwards. Computational analysis of unsteady cavity flows driven by thick shear layers. The Aeronautical Journal, 94(940):355364, 1988.

[68] X. Zhang, A. Rona, and J. A. Edwards. The effect of trailing edge geometry on cavity flow oscillation driven by a supersonic shear layer. The Aeronautical Journal, pages 129-136, March 1998. 